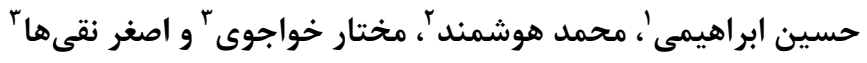

\begin{tabular}{|c|}
\hline 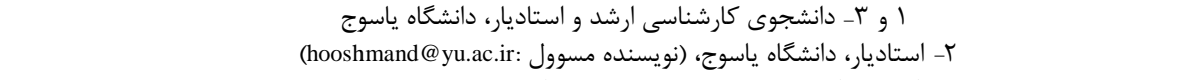 \\
\hline 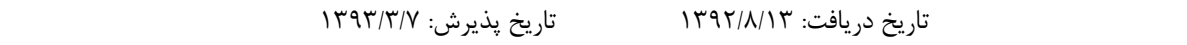 \\
\hline
\end{tabular}

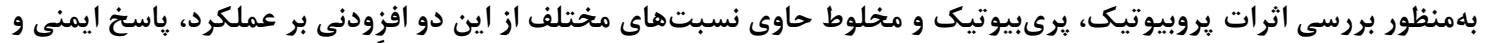

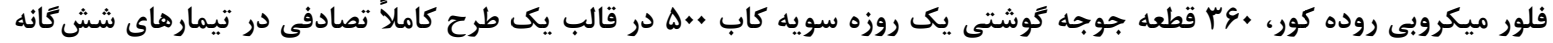

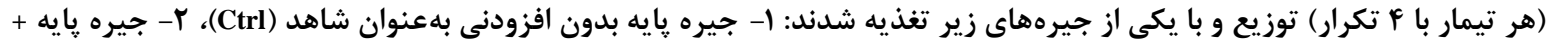

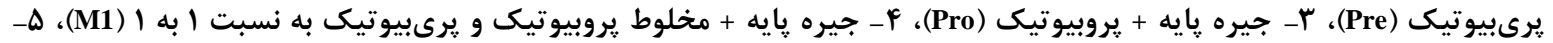

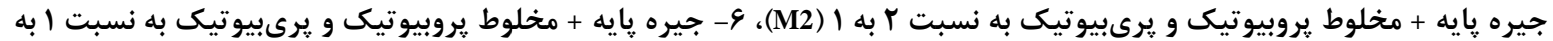

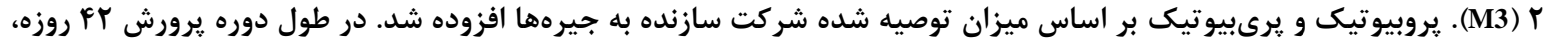

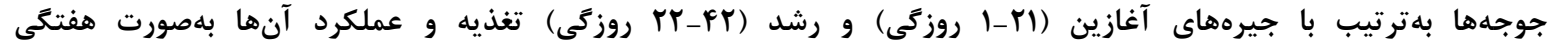

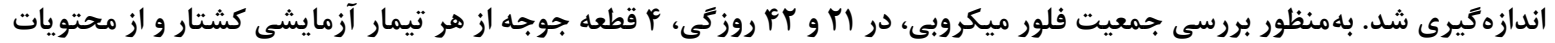

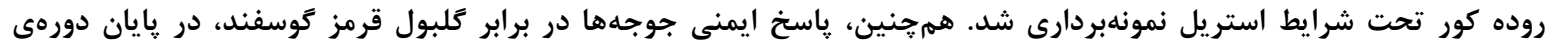

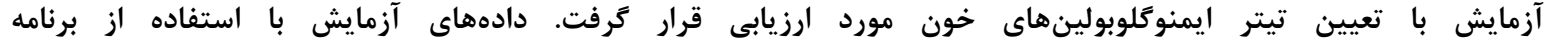

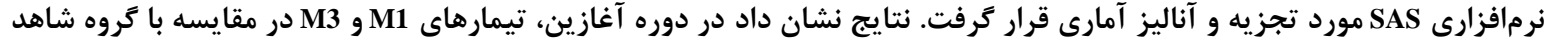

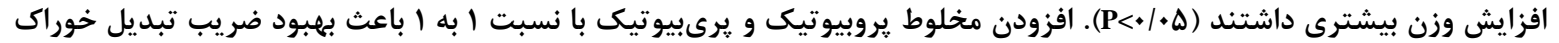

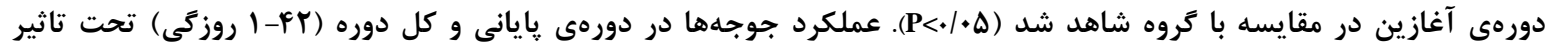

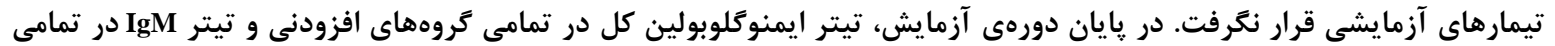

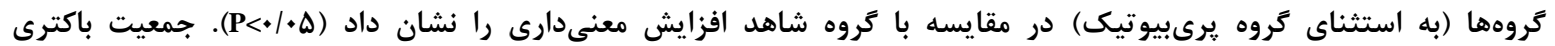

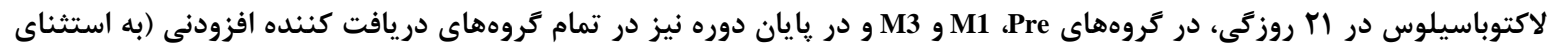

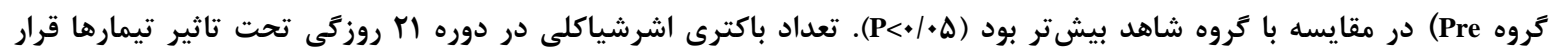

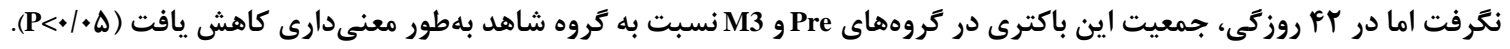

$$
\text { وازههاى كليدى: ياسخ ايمنى، يرىبيوتيك، يروبيوتيك، جوجه توشتى، عملكرد، فلور ميكروبى }
$$

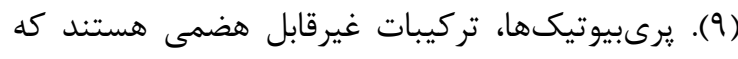

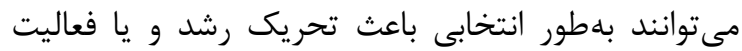

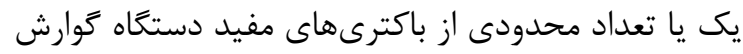

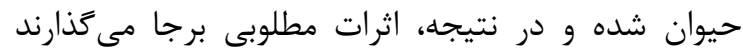

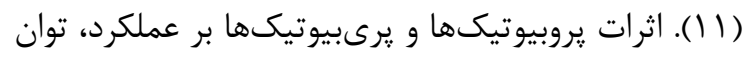

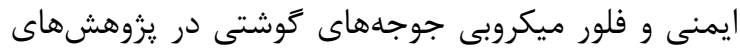

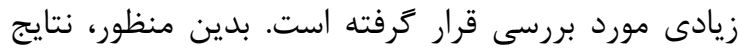

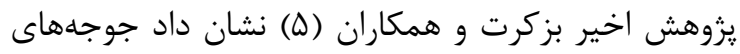

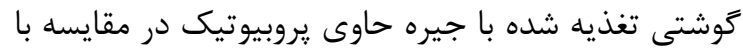

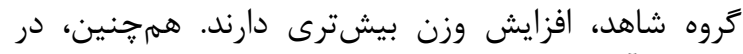

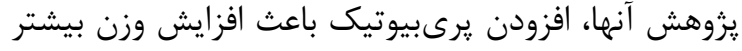

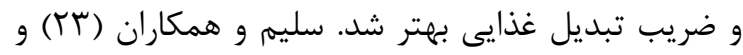

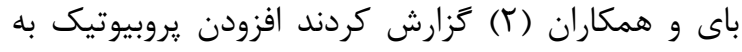

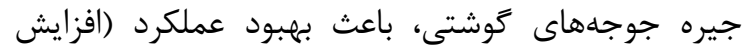

مقدمه - مقه

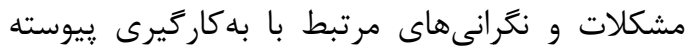

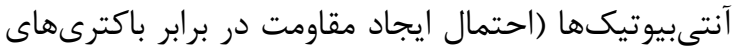

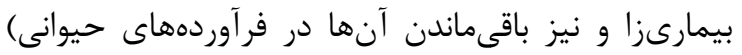

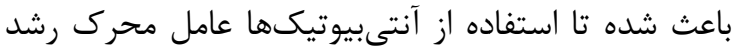

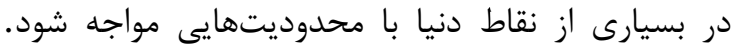

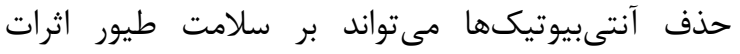

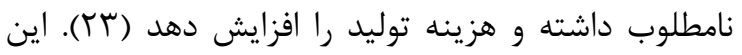

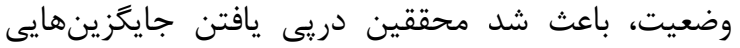

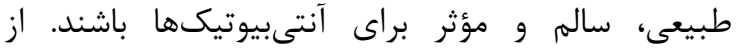

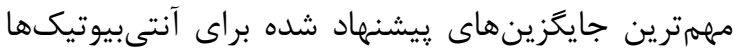

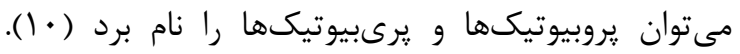

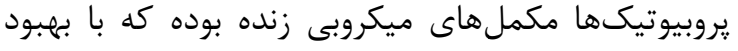

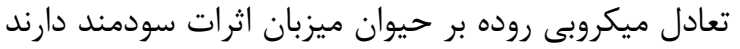


در ايران از زروبيوتيكها و زيرىبيوتيكهاى تجارى

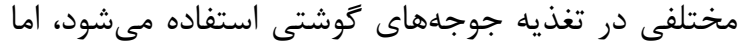

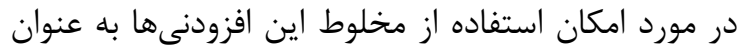

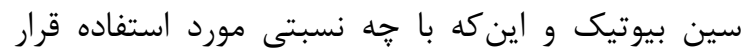

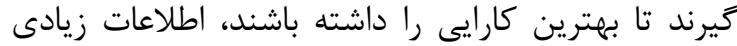

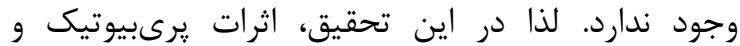

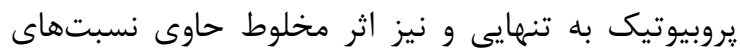

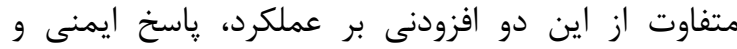

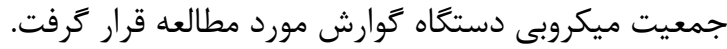

\section{مواد و روشها}

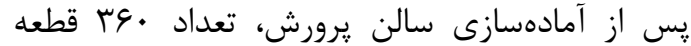

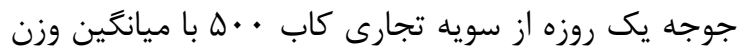

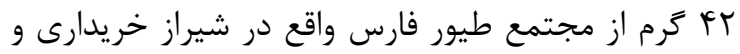

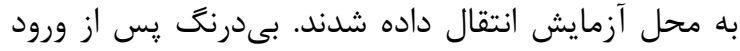

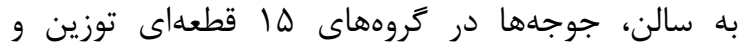

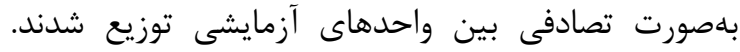

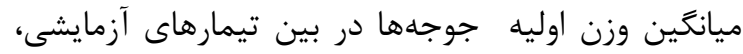

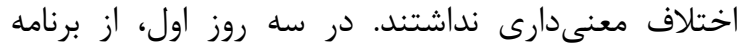

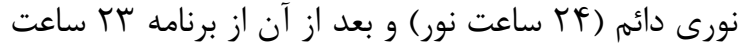

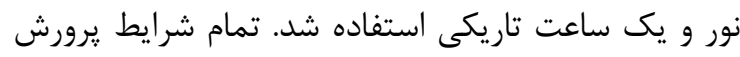

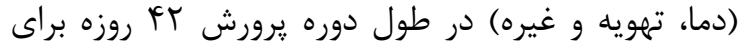

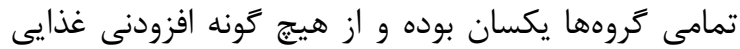

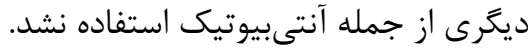

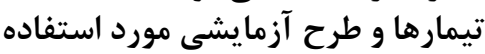

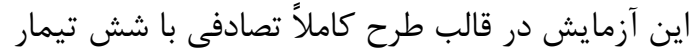

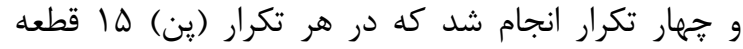

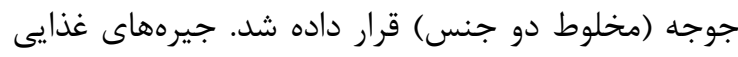

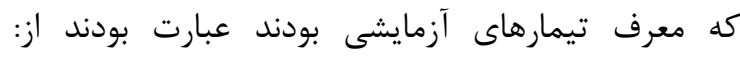

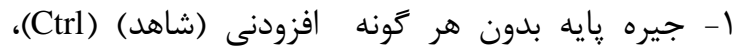

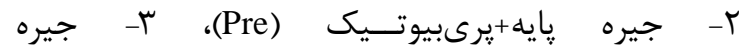

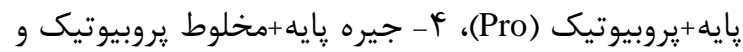

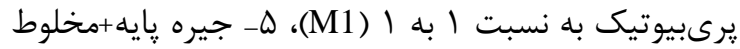

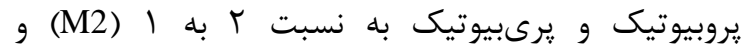

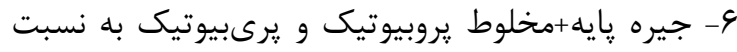

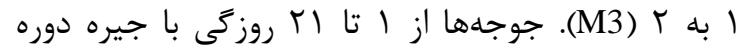

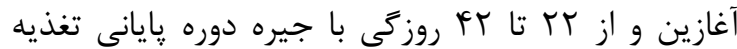

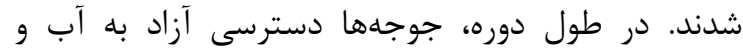

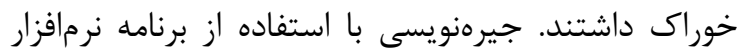
UFFDA

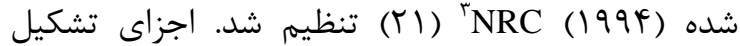

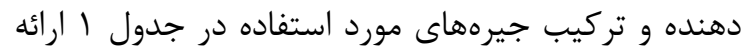

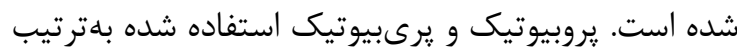

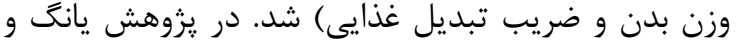

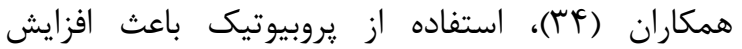

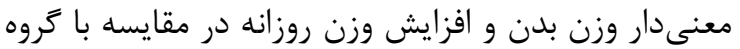
شاهد شد كه اين اثرات سودمند، مشابه اثرات آثرات آنتىبيوتيك بـانيك

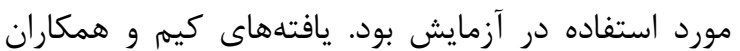

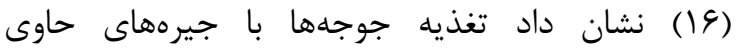

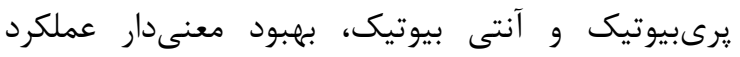
جوجهاى كوشتى را به دنبال دارد.

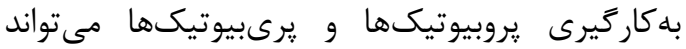

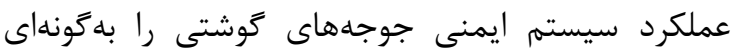

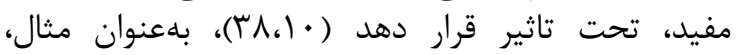

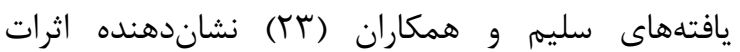

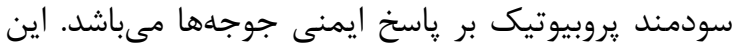

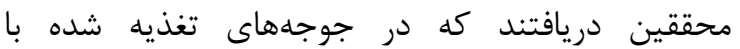

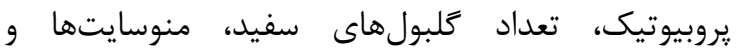

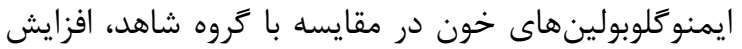

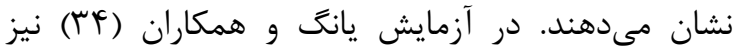

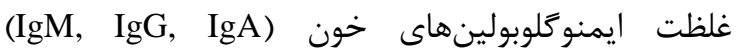

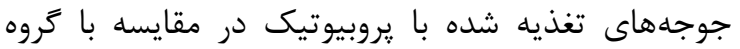

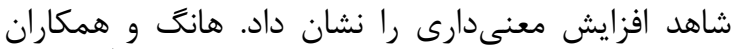

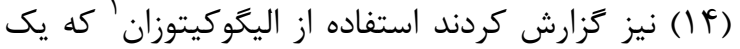

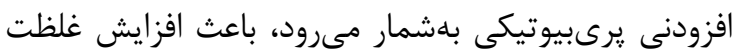

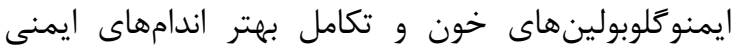

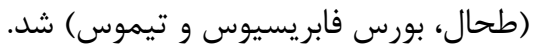

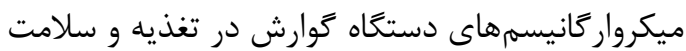

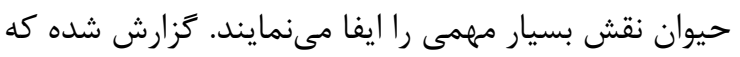

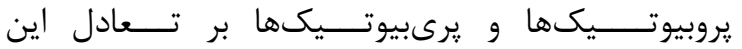

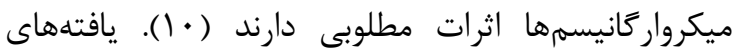

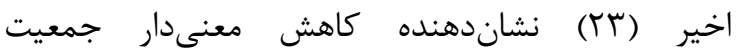

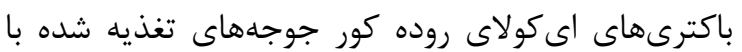

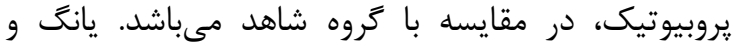

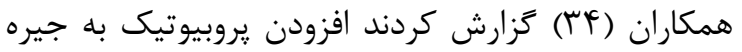

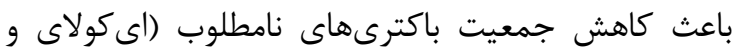

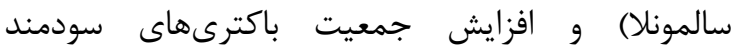

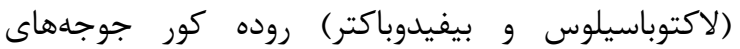

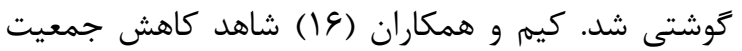

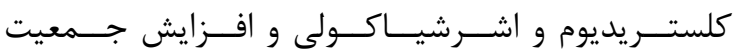

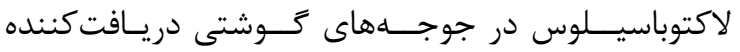
يرى لابيوتيك بودند. مخلوط يروبيوتيك و يرىبيوتيك، سين بينيوتيك ناميده

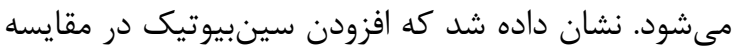

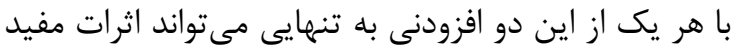

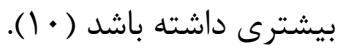
3- National Research Council 


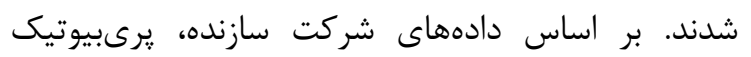

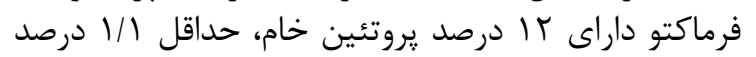

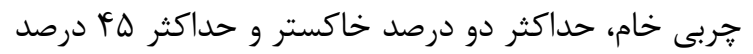
فيبر ميسليوم است.
با نامهاى تجارى : يريمالاك' و فرماكتو'، محصول شركت

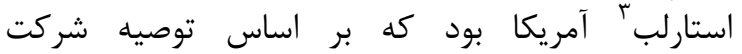

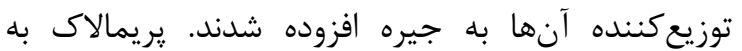

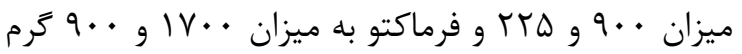

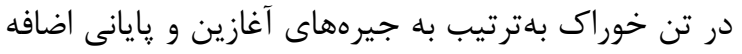

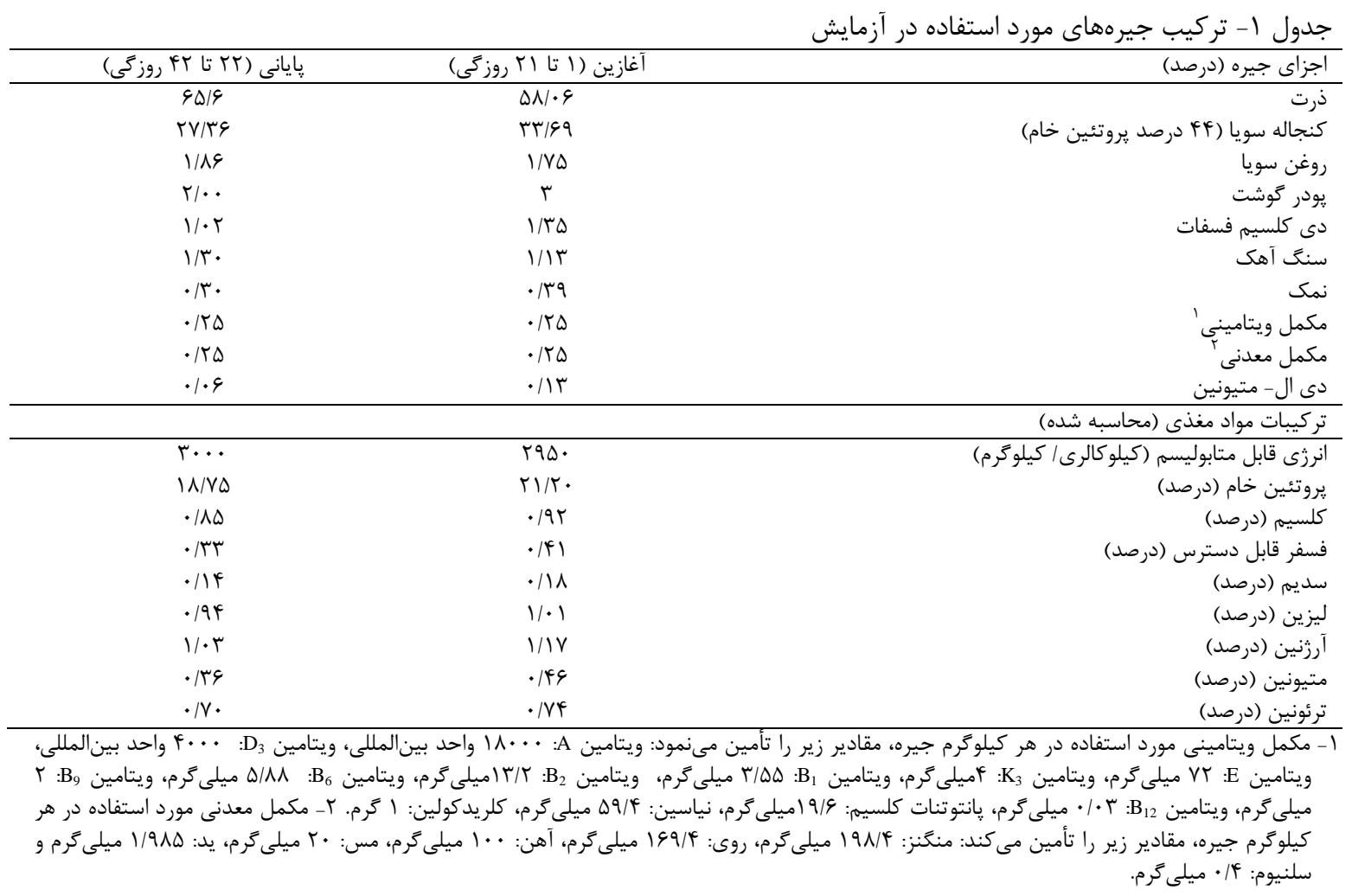

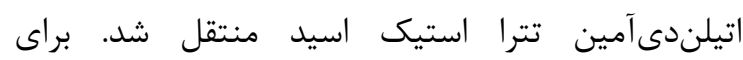

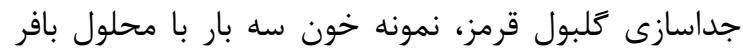

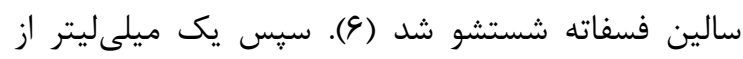

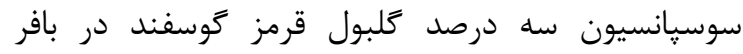

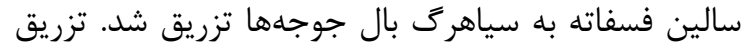

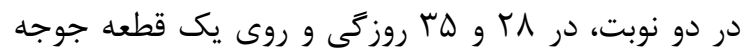

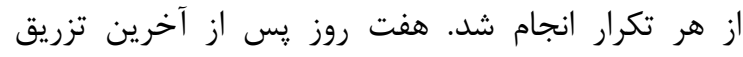

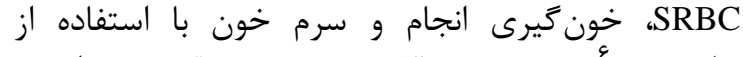

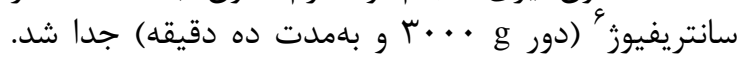

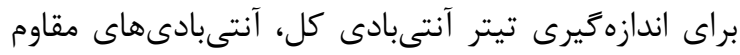

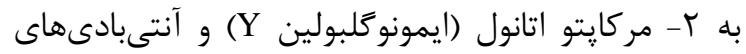

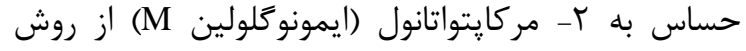

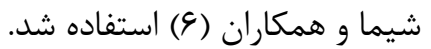

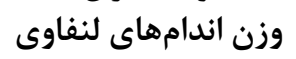

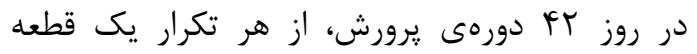

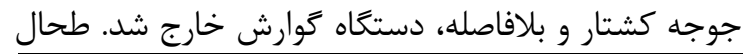

1- Premalac

4- Hemagglutination
جوجههاى هر واحد آزمايشى در پايان هر هفته

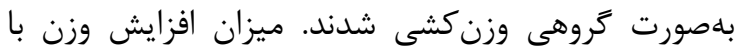

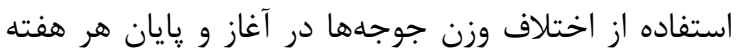

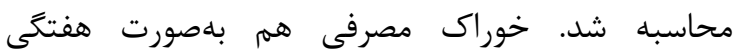

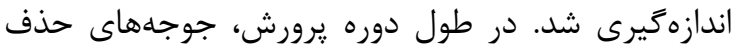

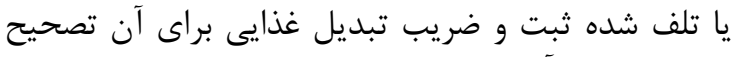

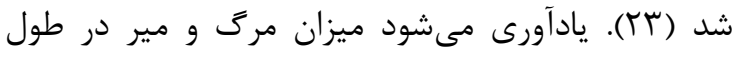

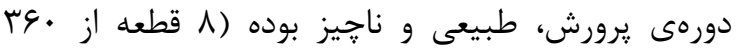

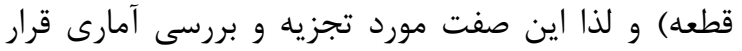

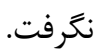
تيتر ايمنو ملوبولين عليه كَلبول قرمز كوسفند

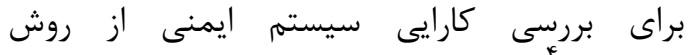

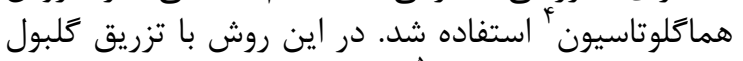

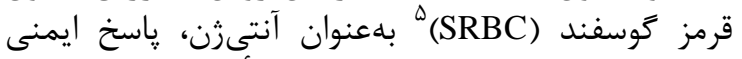

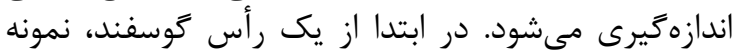

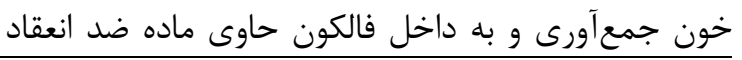


شاهد و افزودنىها اختلاف معنى دارى وجود نداشت، اما

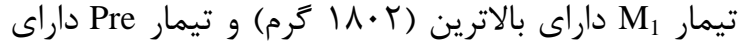

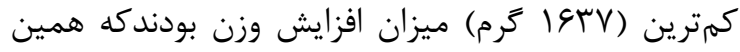

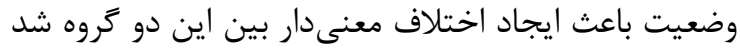

. $(\mathrm{P}<\cdot / \cdot \Delta)$

نتايج مربوط به مصرف خوراك نشان داد در طول

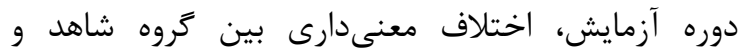

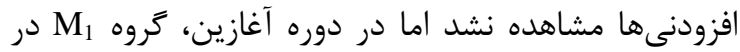

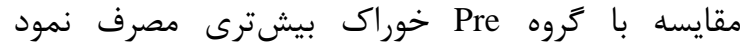

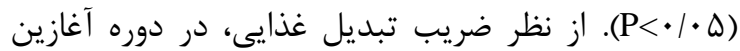

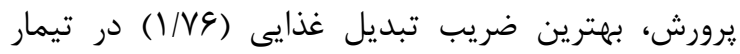

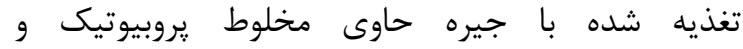

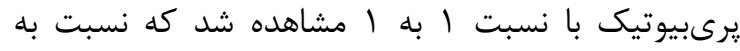

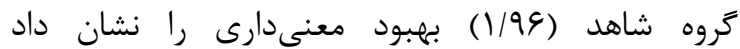
بايانى

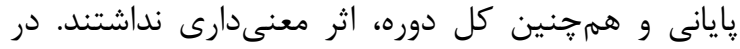

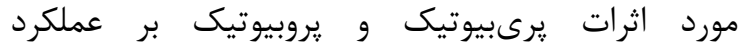

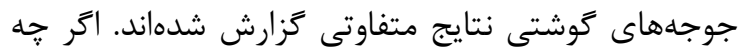

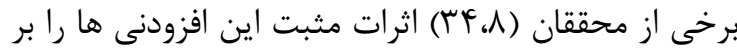

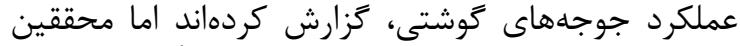

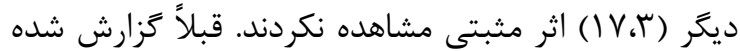

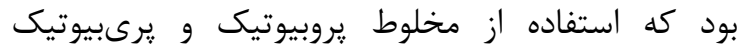

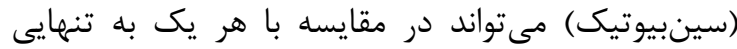

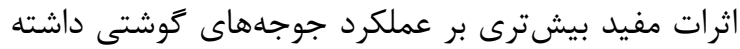

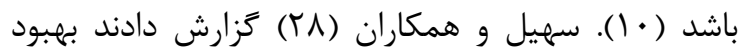

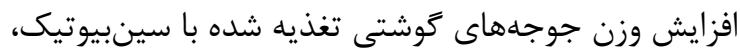

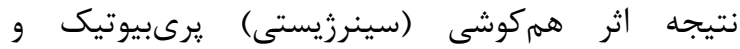

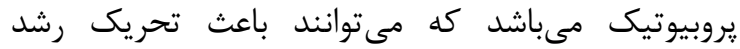

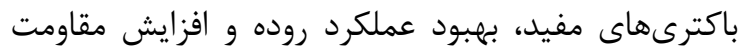

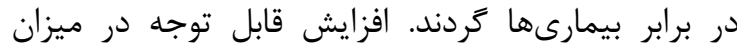

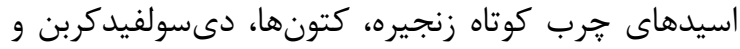

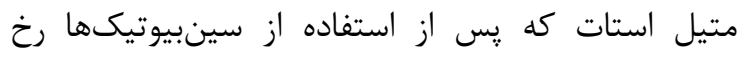

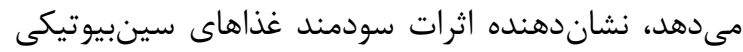

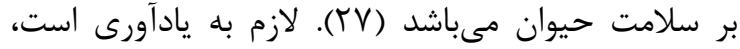

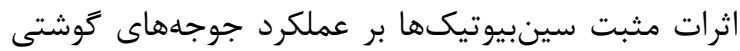

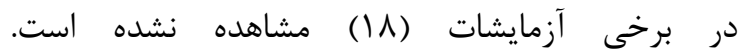

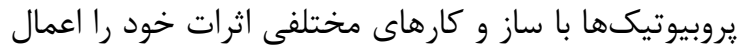

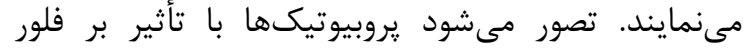

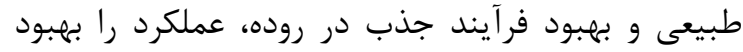

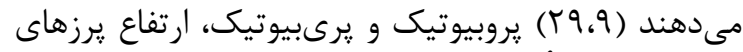

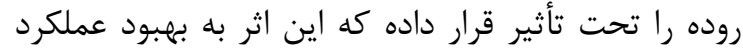

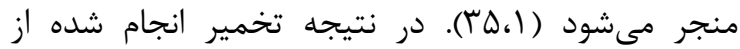

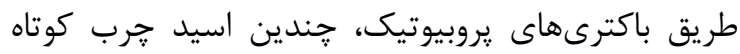

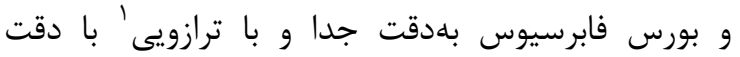

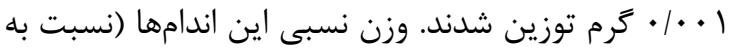

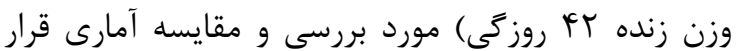

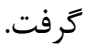
فلور ميكروبى دستخاه كَوارش

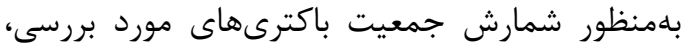

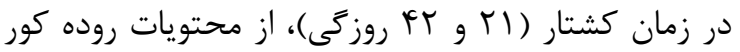

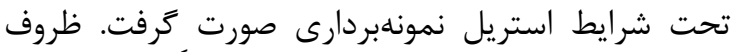

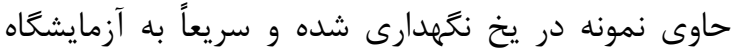

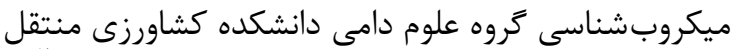

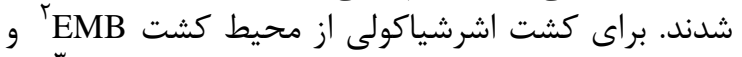

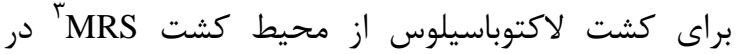

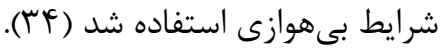

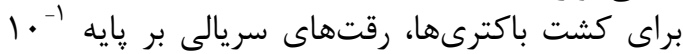

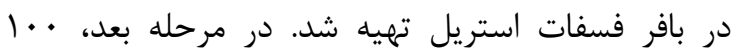

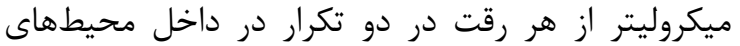

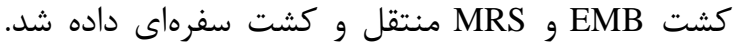

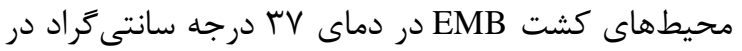

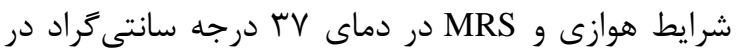

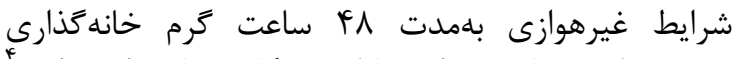

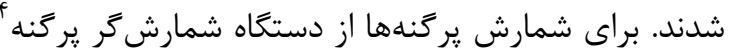

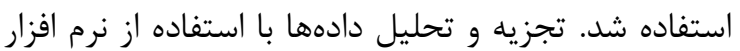

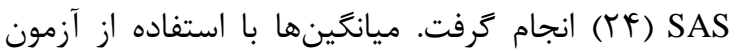
קند دامنهاى دانكن (V) در سطح له درصد مورد مقايسه

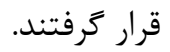

نتايج و بحث

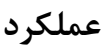

اثر تيمارهاى مختلف آزمايشى بر عملكرد جوجهها

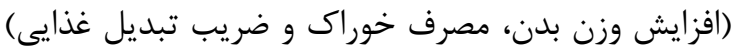

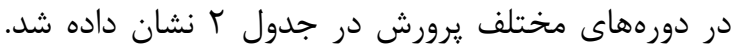

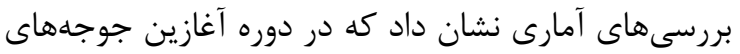

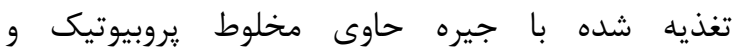

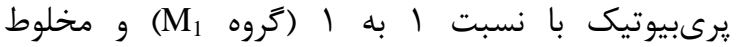

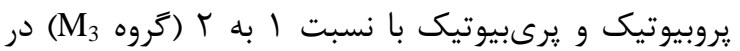

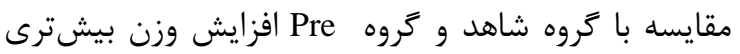

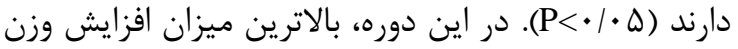

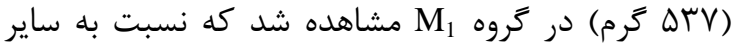

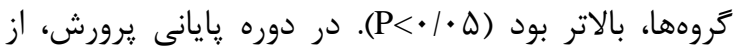

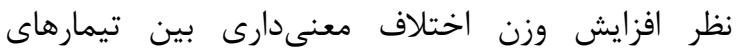

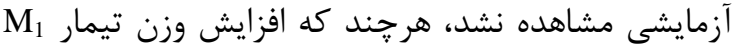

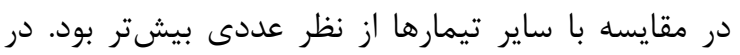

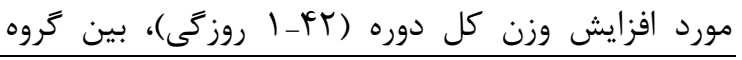
3- De Man, Rogosa and Sharpe 4- Sana SL-902, Iran 


\begin{tabular}{|c|c|c|c|c|c|c|c|}
\hline \multirow{3}{*}{ SEM } & \multicolumn{7}{|c|}{ جدول r- اثر تيمارهاى آزمايشى بر عملكرد جوجههاى گوشتى در دورههاى مختلف يرورش } \\
\hline & \multicolumn{6}{|c|}{ تيمار آزمايشى } & \multirow{2}{*}{ يـارامتر } \\
\hline & M3 & M2 & M1 & Pro & Pre & Ctrl & \\
\hline \multicolumn{8}{|c|}{ افزايش وزن بدن (كَرم) } \\
\hline 11 & $q q r^{D}$ & $|\varphi q|^{\mathrm{bc}}$ & $\Delta r v^{a}$ & $\forall \& q^{D C}$ & $|\nabla \Delta|^{c}$ & $\mu F \Delta^{c}$ & | آ-| روزگى \\
\hline iv & $1 \pi \cdot 4$ & ITIV & 1rG9 & $1 T \cdot \Lambda$ & 1119 & $1 \% \cdot 4$ & 作 \\
\hline is & $1991^{\mathrm{ab}}$ & $19 \mathrm{VV}$ & $1 \Lambda \cdot r^{\mathrm{a}}$ & $19 v v^{a b}$ & $19 \mathrm{rV}^{\mathrm{b}}$ & $|90|^{\mathrm{ab}}$ & \\
\hline \multicolumn{8}{|c|}{ مصرف خوراك (كَم) } \\
\hline Vq & trit & TVDV & $r \Lambda \cdot r$ & r\&19 & TYFF & r\&qV & T \\
\hline 94 & TETr & remf & rVEq & $r V \Delta \cdot$ & 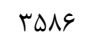 & $r \Delta G \Lambda$ & r \\
\hline \multicolumn{8}{|c|}{ ضريب تبديل غذايى } \\
\hline$\cdot / \cdot 11$ & $T / T \Delta$ & $r / r q$ & $T / T r$ & T/TT & T/MT & T/TF & S \\
\hline$\cdot / \cdot \Delta \Delta$ & T/ו & $r / 1 \Lambda$ & $r / \cdot \Lambda$ & r/T & $r / 19$ & $r / 19$ & \\
\hline
\end{tabular}

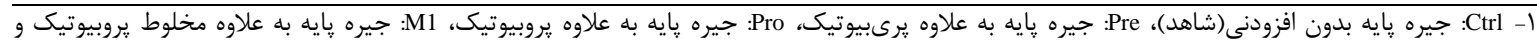

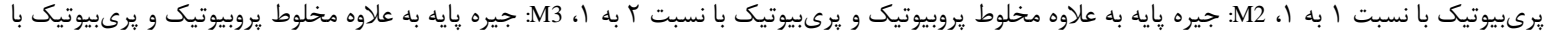

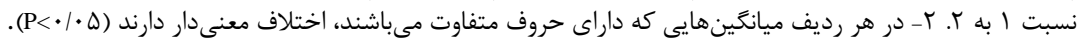

بستخى داشته باشد. محيط برورش، شيوه مديريت، تغذيه،

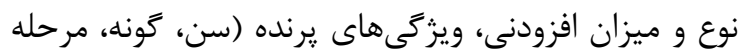

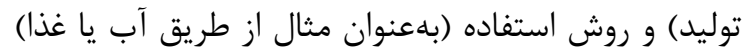

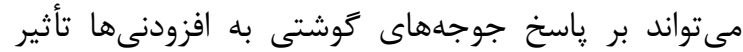

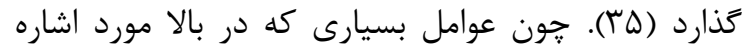

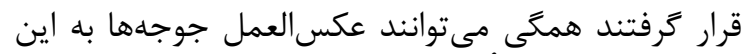

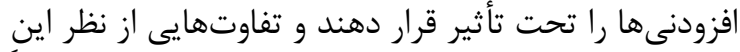

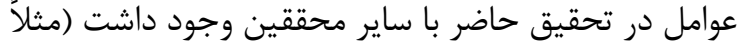

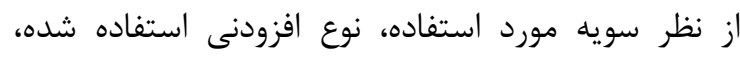

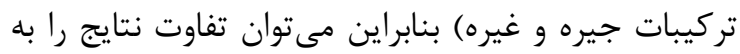

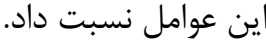

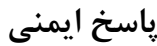

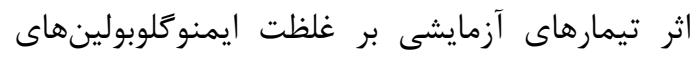

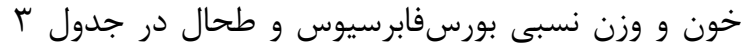

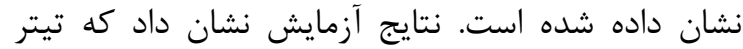

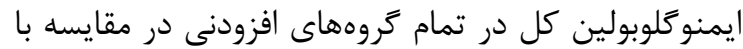

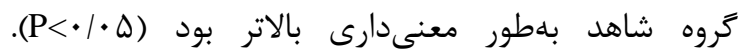

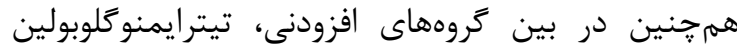
كل در كروه

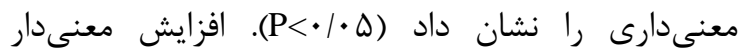

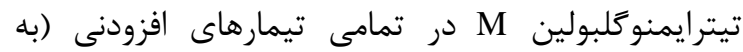

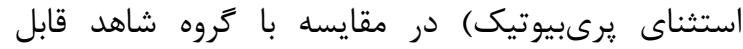

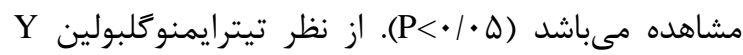

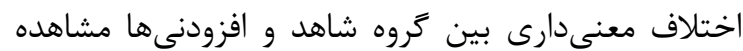

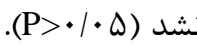

كزارش شده است كه افزودن يرىبيوتيك مىتواند به

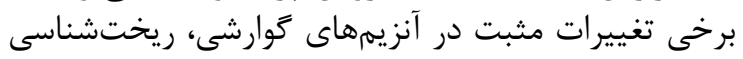

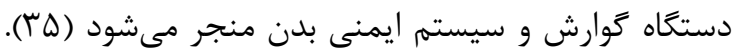

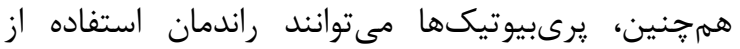

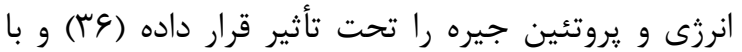

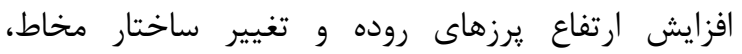

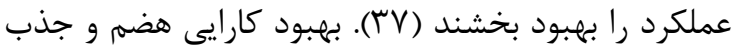

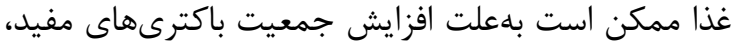

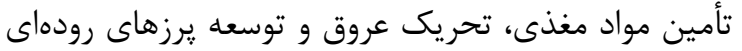

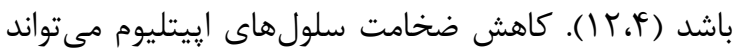

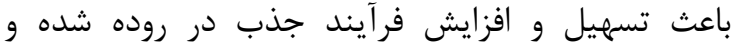

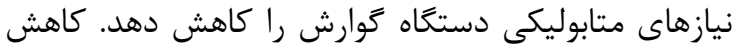

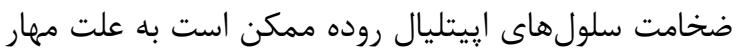

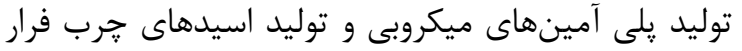

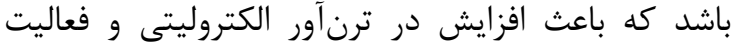

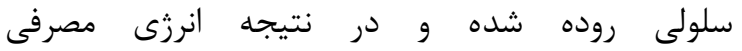

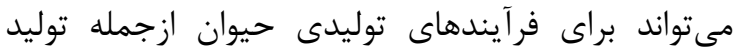

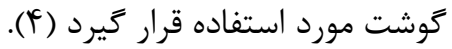

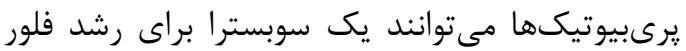

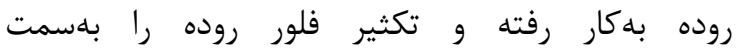

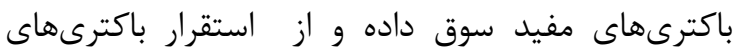
بيمارىزا ممانعت نمايند (•) (1).

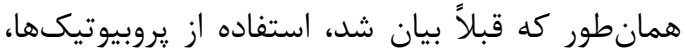

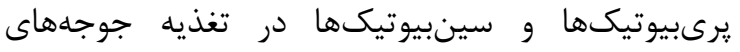

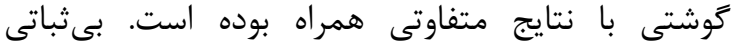
مشاهده شده در نتايج تحقيقات مى تواند به دلايل مختلفى لونى 


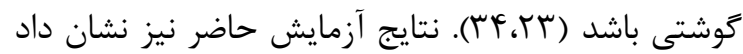

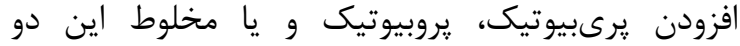

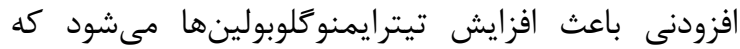

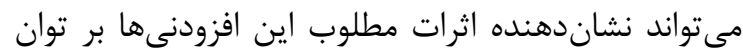

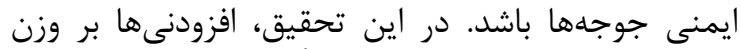

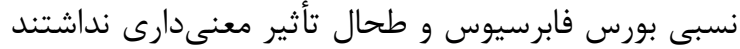

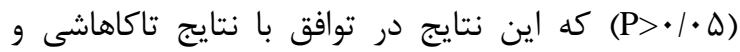

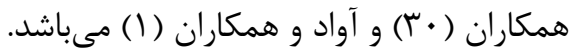

براى ارزيابى سيستم ايمنى طيور از معيارهاى مختلفى إنى

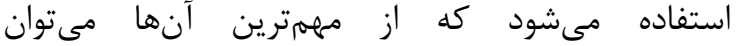

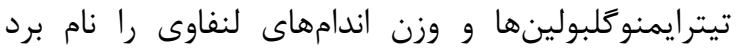

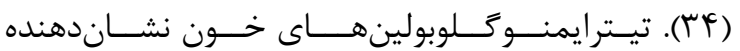

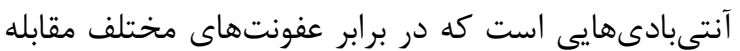

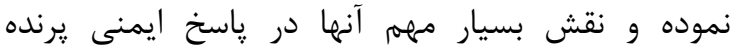

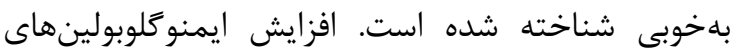

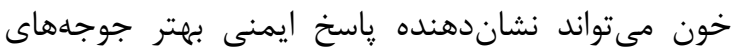

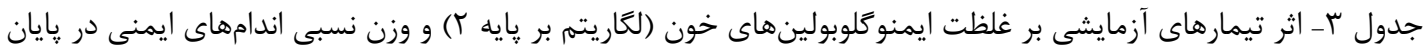

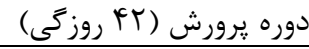

\begin{tabular}{|c|c|c|c|c|c|c|c|}
\hline \multirow{2}{*}{ SEM } & \multicolumn{6}{|c|}{ تيمار آزمايشى } & \multirow[b]{2}{*}{ يـار امتر } \\
\hline & M3 & M2 & M1 & Pro & Pre & Ctrl & \\
\hline \multicolumn{8}{|c|}{ ايمنو لكلوبولينهاى خون } \\
\hline$\cdot \mathrm{NT}$ & $\Delta / .^{\mathrm{a}}$ & $F / r \Delta^{a}$ & $Y / V \Delta^{a}$ & $\Delta / \Delta \cdot{ }^{a}$ & $r / r \Delta^{\mathrm{ab}}$ & $1 / \pi r^{b}$ & $\operatorname{IgM}$ \\
\hline .149 & T/VQ & $r / \cdot$. & $1 / \Delta$ & I/VD & $r / \cdot$. & $r / . \cdot$ & $\operatorname{IgY}$ \\
\hline .195 & $V / V \Delta^{\mathrm{a}}$ & $g / r \Delta^{\mathrm{ab}}$ & $G / r \Delta^{\mathrm{ab}}$ & $V / r \Delta^{a b}$ & $\Delta / r \Delta^{\mathrm{b}}$ & $r / \pi r^{c}$ & كل \\
\hline \multicolumn{8}{|c|}{ وزن اندامهاى ايمنى (درصد وزن بدن) } \\
\hline .1 .19 & $\cdot 119$ & . & $\cdot / 1 T$ &.$/ 19$ & $\cdot / \mathrm{V}$ & $\cdot / 1 r$ & طحال \\
\hline.$/ . Y 4$ & $\cdot 11$. &.$/ 14$ & $\cdot / 11$ & $\cdot 110$ & $\cdot / 11$ & $\cdot / 14$ & بورس فابرسيوس \\
\hline
\end{tabular}

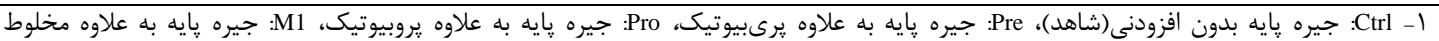

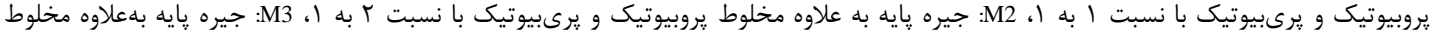

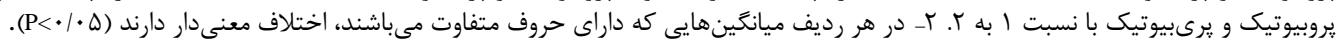

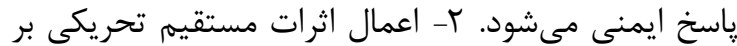

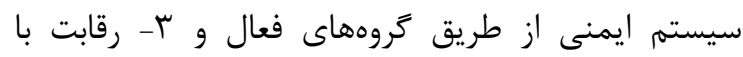

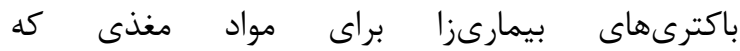

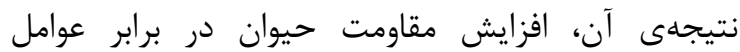

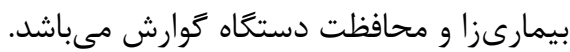

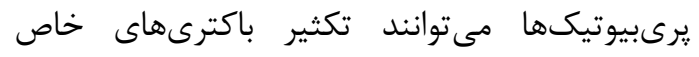

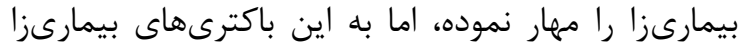

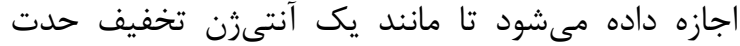

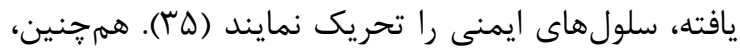

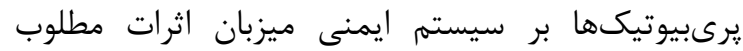

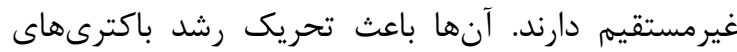

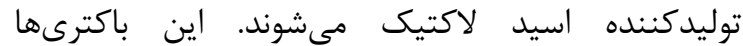

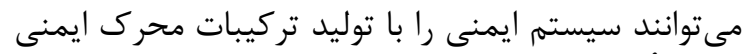

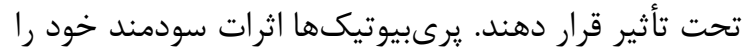

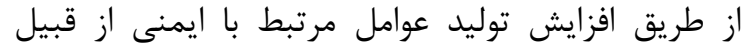

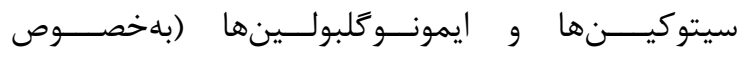

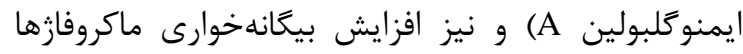

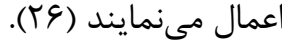
فلور ميكروبى روده كور إنماني اثر تيمارهاى آزمايشى بر كور جمعيت باكترى دهاى رور روده

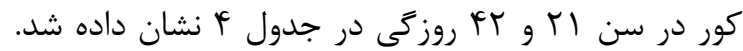

اثرات مثبت يرىبيوتيكها و يروبيوتيكها بر سيسته

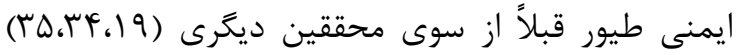

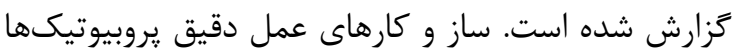

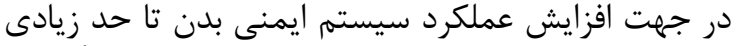

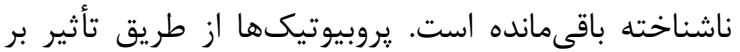

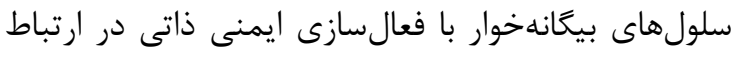

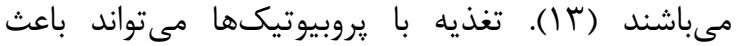

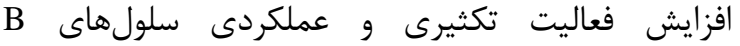

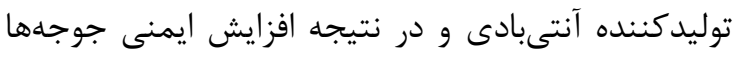

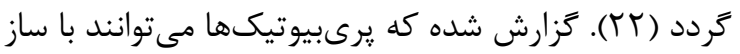

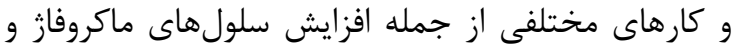

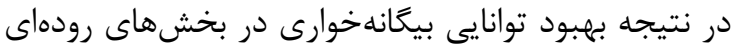

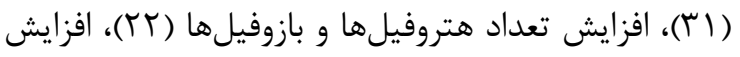

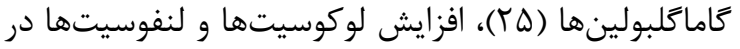

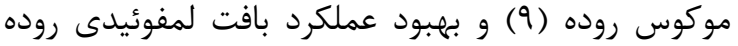

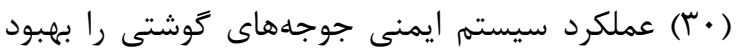

دهند. هانگ و همكاران (f (1)، دلايل احتمالى بهبود مشاهده

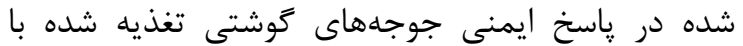

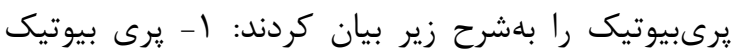

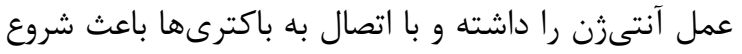




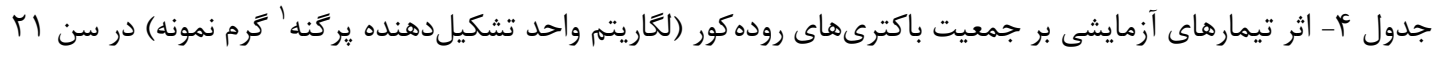
g

\begin{tabular}{|c|c|c|c|c|c|c|c|}
\hline \multirow{2}{*}{ SEM } & \multicolumn{6}{|c|}{ تيمار آزمايشى } & \multirow{2}{*}{ يـارامتر } \\
\hline & M3 & M2 & M1 & Pro & Pre & Ctrl & \\
\hline .1 .9 & $q / v r^{a}$ & $N / \Delta T^{c d}$ & $9 / \cdot r^{\mathrm{bc}}$ & $N / G Y^{\mathrm{cd}}$ & $9 / \pi 1^{\text {ab }}$ & $N / \psi^{4}$ & لاكتو باسيلوس \\
\hline . $/ V V$ & $9 / \kappa \Lambda^{a}$ & $9 / / \Lambda^{a}$ & $\mathrm{q} / 1 \mathrm{~V}^{\mathrm{a}}$ & $q / \cdot r^{a}$ & $\Lambda / \cdot 9^{\mathrm{ab}}$ & $V / \& \varepsilon^{D}$ & اشرشياكولى \\
\hline $\begin{array}{l}. k \Delta \\
. / 4 \Delta\end{array}$ & $\begin{array}{l}V / 9 T \\
q / T \cdot b c\end{array}$ & $\begin{array}{l}V / \Delta T \\
V / Q T^{\mathrm{a}}\end{array}$ & $\begin{array}{l}V / F \Delta \\
V / r \Lambda^{a}\end{array}$ & $\begin{array}{l}9 / 90 \\
9 / 91^{\text {ab }}\end{array}$ & $\begin{array}{l}V / G Y^{q} \\
\Delta / V q^{c}\end{array}$ & $\begin{array}{l}q / 9 \varphi \\
V / \Delta f^{a}\end{array}$ & 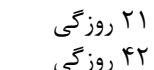 \\
\hline
\end{tabular}

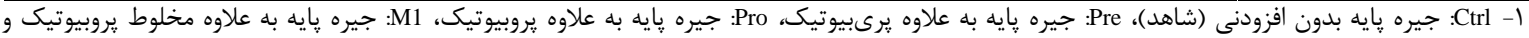

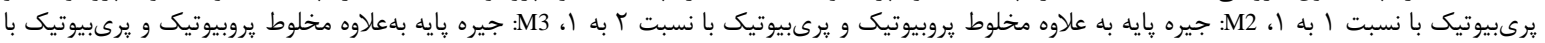

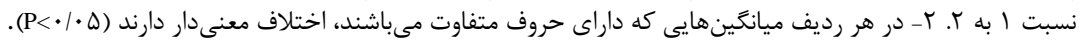

كربوهيدراتها مقدار زيادى لاكتات توليد مى كنند. اين

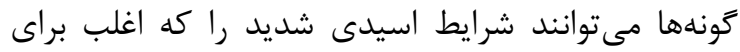

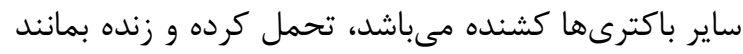

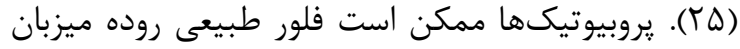

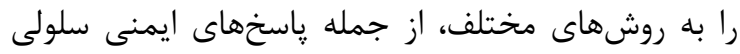

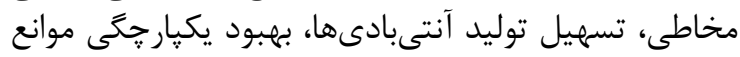

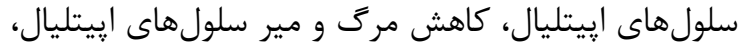

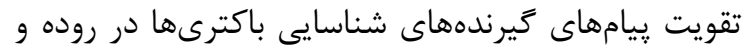

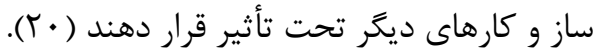

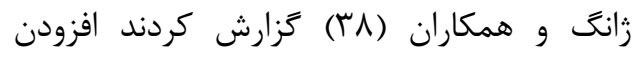

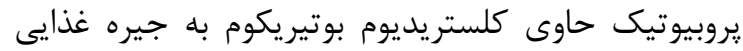

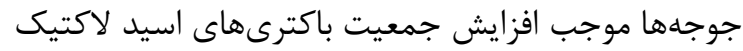

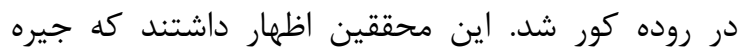

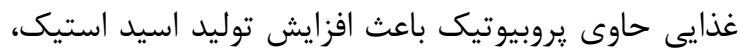

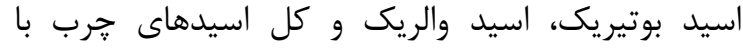

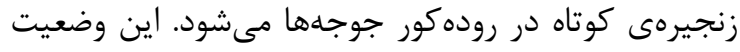

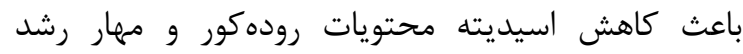

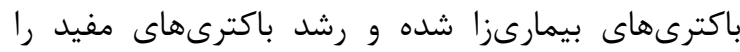

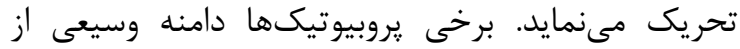

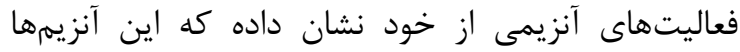

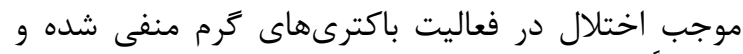

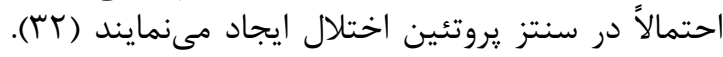

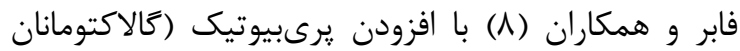

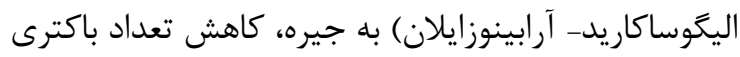

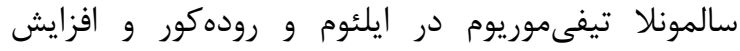

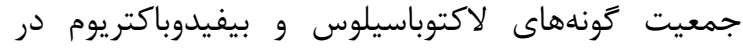
رودهور

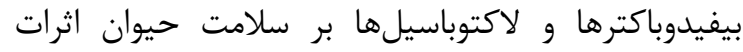

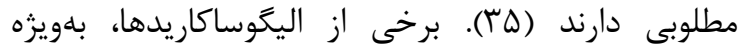
ماناناليكوساكاريدها، ممكن است بلهطور الئ مستقيه از اتصال
نتايج نشان داد تعداد ير گَنههاى باكترى لاكتوباسيلوس

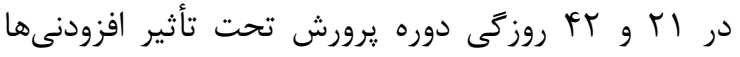

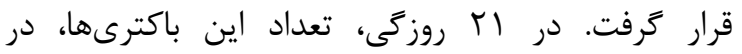

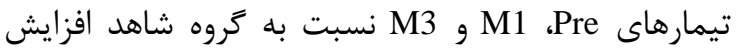

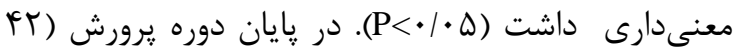

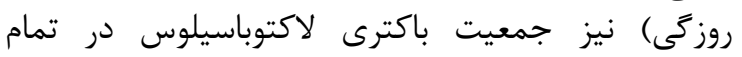

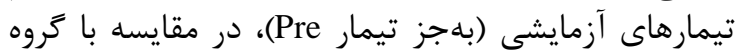

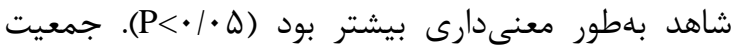

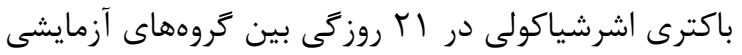

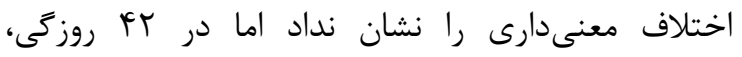

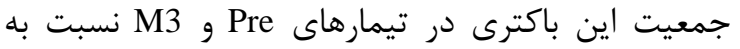

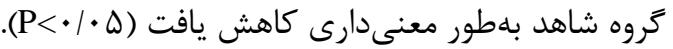

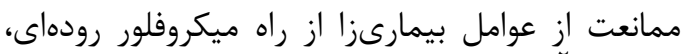

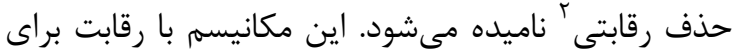

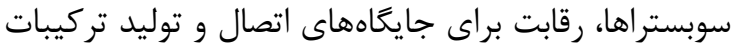

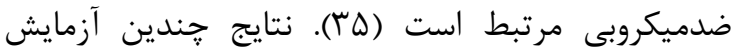

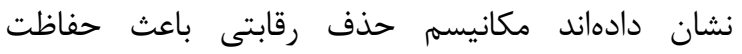

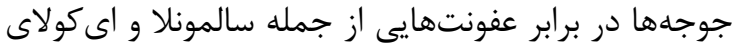

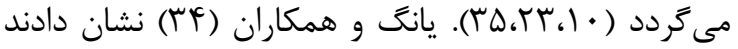

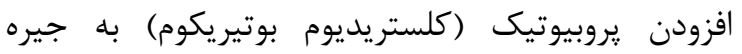

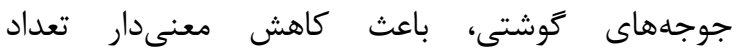

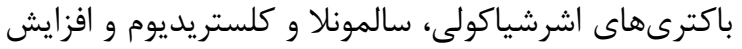

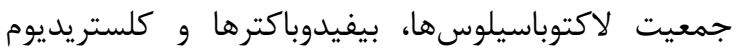

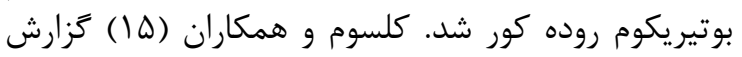

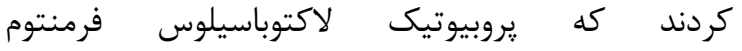

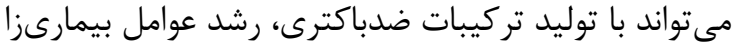

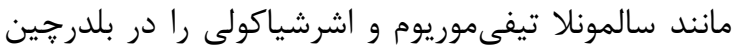

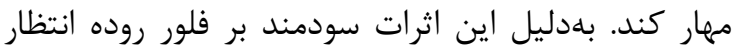
مىرود عملكرد بهبود يابد. كونههاى لاكتوباسيلوس احتمالاً با استفاده از 


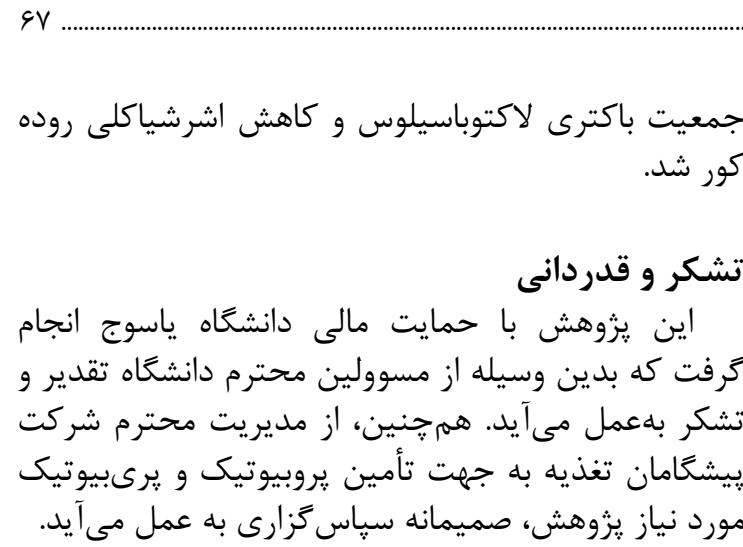

يثوهشهاى توليدات دامى سال هفتم/ شماره س |/ بهار و تابستان هوس|

باكترىهاى بيمارىزا به سلولهاى اييتليال روده كوجى نه

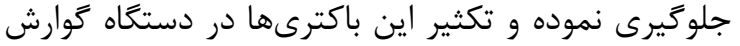
را مهار نمايند (·) (1).

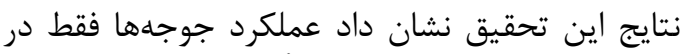

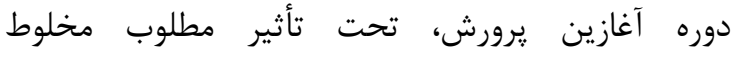

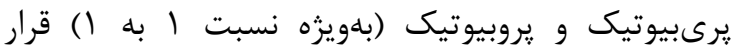

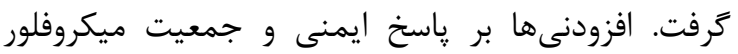

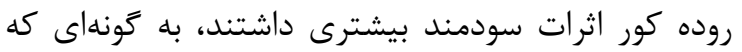

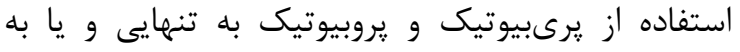

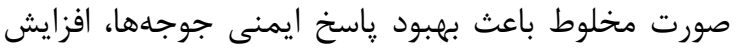

منابع

1. Awad, W.A., K. Ghareeb, S. Abdel-Raheem and J. Böhm. 2009. Effects of dietary inclusion of probiotic and synbiotic on growth performance, organ weights, and intestinal histomorphology of broiler chickens. Poultry Science, 88: 49-55.

2. Bai, S.P., A.M. Wu, X.M. Ding, Y. Lei, J. Bai, K.Y. Zhang and J.S. Chio. 2013. Effects of probioticsupplemented diets on growth performance and intestinal immune characteristics of broiler chickens. Poultry Science, 92: 663-670.

3. Baurhoo, B., F. Goldflus and X. Zhao. 2009. Purified cell wall of Saccharomyces cerevisiae increases protection against intestinal pathogens in broiler chickens. International Journal of Poultry Science, 8: 133-137.

4. Bedford, M. 2000. Removal of antibiotic growth promoters from poultry diets: Implications and strategies to minimize subsequent problems. World's Poultry Science Journal, 56: 347-365.

5. Bozkurt, M., N. Aysul, K. Kucukyilmaz, S. Aypak, G. Ege, A. U. Catli, H. Akşit, F. Coven, K. Seyrek, and M. Cinar. 2014. Efficacy of in-feed preparations of an anticoccidial, multienzyme, prebiotic, probiotic and herbal essential oil mixture in healthy and Eimeria spp.-infected broilers. Poultry Science, 93: 389399.

6. Cheema, M.A., M.A. Qureshi and G.B. Havenstein. 2003. A comparison of the immune response of a 2001 commercial broiler with a 1957 random bred broiler strain when fed representative 1957 and 2001 broiler diets. Poultry Science, 82: 1519-1529.

7. Duncan, D.B. 1955. Multiple range tests and multiple F test. Biometrics, 11:1-42.

8. Faber, T.A., R.N. Dilger, M. Iakiviak, A.C. Hopkins, N.P. Price and Jr, G.C. Fahey. 2012. Ingestion of a novel galactoglucomannan oligosaccharide-arabinoxylan (GGMO-AX) complex affected growth performance and fermentative and immunological characteristics of broiler chicks challenged with Salmonella typhimurium. Poultry Science, 91: 2241-2254.

9. Fuller, R. 1989. "Probiotics in man and animals. Journal of Applied Bacteriology, 66: 365-378.

10. Gaggia, F., P. Mattarelli and B. Biavati. 2010. Probiotics and prebiotics in animal feeding for safe food production. International Journal of Food Microbiology, 141: 15-28.

11. Gibson, G.R. and M.B. Roberfroid. 1995. Dietary modulationof the human colonic microbiota: introducing the concept of prebiotics. Journal of Nutrition, 125: 1401-1412.

12. Gilmore, M.S. and J.J. Ferretti. 2003. The thin line between gut commensal and pathogen. Science, 299: 1999-2002.

13. Higgins, S.E., G.F. Erf, J.P. Higgins, S.N. Henderson, A.D. Wolfenden, G. Gaona-Ramirez and B.M. Hargis. 2007. Effect of probiotic treatment in broiler chickens on intestinal macrophage numbers and phagocytosis of salmonella enteritidis by abdominal exudate cells. Poultry Science, 86: 2315-2321.

14. Huang, R.L., Z.Y. Deng, C. Yang, Y.L. Yin, M.Y. Xie, G.Y. Wu, T.J. Li, L.L. Li, Z.R. Tang, P. Kang, Z.P. Hou, D. Deng, H. Xiang, X. Feng Kong and Y.M. Guo. 2007. Dietary oligochitosan supplementation enhances immune status of broilers. Journal of the Science of Food and Agriculture, 87: 153-159.

15. Kalsum, U., H. Soetanto Achmanu and O. Sjofjan. 2012. Influence of a probiotic containing Lactobacillus fermentum on the laying performance and egg quality of Japanese quails. International Journal of Poultry Science, 11: 311-315.

16. Kim, G.B., Y.M. Seo, C.H. Kim and I.K. Paik. 2011. Effect of dietary prebiotic supplementation on the performance, intestinal microflora and immune response of broilers. Poultry Science, 90: 75-82.

17. Lee, K.W., S.H. Lee, H.S. Lillehoj, G.X. Li, S.I. Jang, U.S. Babu, M.S. Park, D.K. Kim, E.P. Lillehoj, A.P. Neumann, T.G. Rehberger and G.R. Siragusa. 2010. Effects of direct-fed microbials on growth performance, gut morphometry, and immune characteristics in broiler chickens. Poultry Science, 89: 203216.

18. Midilli, M., M. Alp, N. Kocabaglh, O.H. Muglali, N. Turan, H. ilmaz and S. Cakir. 2008. Effects of dietary probiotic and prebiotic supplementation on growth the performance and serum IgG cocentaration of broilers. Journal of Animal Science, 38: 21-27. 
19. Naseri Alavi, A., A. Zakeri, B. Kamrani and Y. Pourakbari. 2012. Effect of prebiotics, probiotics, acidfire, growth promoter antibiotics and synbiotic on humural immunity of broiler. Global Veterinaria, 8: 612617.

20. Ng, S.C., A.L. Hart, M.A. Kamm, A.J. Stagg and S.C. Knight. 2009. Mechanisms of action of probiotics: Recent advances. Inflammatory Bowel Diseases, 15: 300-310.

21. NRC. 1994. Nutrient Requirements of Poultry. National Academy Press, Washington, DC.

22. Panda, A.K., M.R. Reddy, S.V. RamaRao, M.V.L.N. Raju and N.K. Paraharaj. 2000. Growth, carcass characteristics, immune competence and response to Escherchia coli on broiler fed diets with various level of probiotic. Archive fur Geflugelkunde, 64: 152-156.

23. Salim, H.M., H.K. Kang, N. Akter, D.W. Kim, J.H. Kim, M.J. Kim, J.C. Na, H.B. Jong, H.C. Choi, O.S. Suh and W.K. Kim. 2013. Supplementation of direct-fed microbials as an alternative to antibiotic on growth performance, immune response, cecal microbial population and ileal morphology of broiler chickens. Poultry Science, 92: 2084-2090.

24. SAS. 2005. User's Guide. Version 9. SAS Institute, Cary, NC.

25. Shashidhara, R.G. and G. Devegowda. 2003. Effect of dietary mannan oligosaccharide on broiler breeder production traits and immunity. Poultry Science, 82: 1319-1325.

26. Silva, V.K., J. Della Torre da Silva, K.A.A. Torres, D E. De Faria Filho, F. Hirota Hada and V.M. Barbosa De Moraes. 2009. Humoral immune response of broilers fed diets containing yeast extract and prebiotics in the prestarter phase and raised at different temperatures. Journal of Applied Poultry Research, 18: 530540.

27. Singh Sekhon, B. and S. Jairath. 2010. Prebiotics, probiotics and synbiotics: an overview. Journal of Pharmaceutical Education and Research, 2: 13-36.

28. Sohail, M.U., M.E. Hume, J.A. Byrd, D.J. Nisbet, A. Ijaz, A. Sohail, M.Z. Shabbir and H. Rehman. 2012. Effect of supplementation of prebiotic mannan-oligosaccharides and probiotic mixture on growth performance of broilers subjected to chronic heat stress. Poultry Science, 91: 2235-2240.

29. Sohail, M.U., Z.U. Rahman, A. Ijaz, M.S. Yousaf, K. Ashraf, T. Yaqub, H. Zenab, H. Anwar and H. Rehman. 2011. Single or combined effects of mannan-oligosaccharides and probiotics supplements on the total oxidants, total antioxidants, enzymatic antioxidants, liver enzymes and serum trace minerals in cyclic heat stressed broilers. Poultry Science, 90: 2573-2577.

30. Takahashi, S.E., A.A. Mendes, E.S.P.B. Saldanha, C.C. Pizzolante, K. Pelícia, R.R. Quinteiro, C.M. Komiyama, R.G. Garcia and I.C.L. Almeida Paz. 2005. Efficiency of prebiotics and probiotics on the performance, yield, meat quality, and presence of Salmonella spp in carcasses of free-range. Brazilian Journal of Poultry Science, 7: 151-157.

31. Tellez, G., G. Nava, J.L. Vicente, A.M. Donghue, W.E. Huff, J. Balog, D.J. Donoghue, L.M. Sutton, S. Higgins and B.M. Hargis. 2002. Evaluation of the effect of dietary Aspergillus sp. Meal prebiotic (Fermacto) on pullet performance, intestinal strength, tibial diameter and tibial strength: Hatch to 30 days of age. Poultry Science, 83: 60 (Abstract).

32. Vila, B., E. Esteve-Garcia and J. Brufau. 2010. Probiotic micro organisms: 100 years of innovation and efficacy; modes of action. World's Poultry Science Journal, 65: 369-380.

33. Wong, J.M.W., R. De Souza, C.W.C. Kendall, A. Emam and D.J.A. Jenkins, 2006. Colonic health: Fermentation and short chain fatty acids. Journal of Clinical Gastroenterology, 40: 235-243.

34. Yang, C.M., G.T. Cao, P.R. Ferket, T.T. Liu, L. Zhou, L. Zhang, Y.P. Xiao and A.G. Chen. 2012. Effects of probiotic, Clostridium butyricum, on growth performance, immune function, and cecal microflora in broiler chickens. Poultry Science, 91: 2121-2129.

35. Yang, Y., P.A. Iji and M. Choct. 2009. Dietary modulation of gut microflora in broiler chickens: a review of the role of six kinds of alternatives to in-feed antibiotics. World's Poultry Science, 65: 97-114.

36. Yang, Y., P.A. Iji, A. Kocher, E. Thomson, L.L. Mikkelsen and M. Choct. 2008. Effects of mannanoligosaccharide in broiler chicken diets on growth performance, energy utilisation, nutrient digestibility and intestinal microflora. British Poultry Science, 49: 186-194.

37. Yang, Y., P.A. Iji and M. Choct. 2007. Effects of different dietary levels of mannanoligosaccharide on growth performance and gut development of broiler chickens. Asian-Australasian Journal of Animal Science, 20: 1084-1091.

38. Zhang, B., X. Yang, Y. Guo and F. Long. 2011. Effects of dietary lipids and Clostridium butyricum on the performance and the digestive tract of broiler chickens. Archive of Animal Nutrition, 65: 329-339. 


\title{
Single or Combined Effects of Prebiotic and Probiotic on Performance, Immunity Response and Gut Flora of Broiler Chickens
}

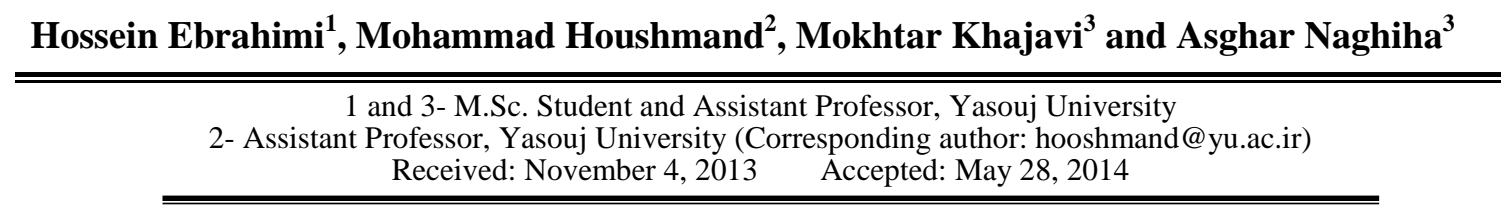

\begin{abstract}
In order to investigate the effects of probiotic, prebiotic and a mixture of these feed additives (at different ratio) on the performance, immunity response and cecal microflora of broiler chickens, in a complete random design, a total of 360 ond-day-old broiler chicks (Cobb 500) were randomly distributed among 6 experimental treatments with four replicates. Experimental treatments (diets) were: 1- a basal diet without any additive, as control (Ctrl) 2- basal diet added with prebiotic (Pre) 3- basal diet added with probiotic (Pro) 4- basal diet added with a mixture of probiotic and prebiotic at ratio of 1:1 (M1), 5- basal diet added with a mixture of probiotic and prebiotic at ratio of 2:1 (M2), 6- basal diet added with a mixture of probiotic and prebiotic at ratio of 1:2 (M3). Probiotic and prebiotic were added to diets in amounts as recommended by producer. During $42 \mathrm{~d}$ rearing period, birds were fed starter and grower diets form 1 to 21 and 22 to 42 days of age, respectively. Performance traits were measured weekly. On d 21 and 42, one bird from each replicate pen, was slaughtered and cecal content was collected, under sterile condition, to determine the E. coli and Lactobacillus count. In addition, immunity respose to SRBC was measured at the end of the experiment. Data were subjected to one way analysis of variance, by SAS sotware. The results indicated that in the starter phase, birds fed diets M1 or M3 achieved more gain compared to the control group $(\mathrm{P}<0.05)$. At the same time, feeding with M1 diet resulted in a better FCR than the control group $(\mathrm{P}<0.05)$. Significant differences were not observed for performance traits in the finisher phase or whole period (1-42 d of age) of the experiment. All supplemented diets had more total antibody titer against SRBC compared to the control group, at the end of the experiment $(\mathrm{P}<0.05)$. Also, IgM titer was higher in all supplemented treatments (except for Pre) relative to the control group $(\mathrm{P}<0.05)$. On d 21, lactobacillus number was higher in Pre, M1 and M3 treatments than the control $(\mathrm{P}<0.05)$. Also, at the end of the experiment, all supplemented tretments (except for Pre) had higher count of lactobasillus compared to the control group $(\mathrm{P}<0.05)$. On $\mathrm{d} 21$, E. coli count was significantly not influenced by experimental treatments, but significant reductions in E. coli content were observed for Pre and M1 treatments compared to the control group at $42 \mathrm{~d}$ of age $(\mathrm{P}<0.05)$.
\end{abstract}

Keywords: Broiler, Gut microflora, Immunity response, Prebiotics, Performance, Probiotics 